\title{
Твердотельные СВЧ-переключатели средней и большой мощности
}

\author{
Часть 2
}

\begin{abstract}
В первой части статьи, опубликованной в восьмом номере журнала «ЭЛЕКТРОНИКА: Наука, Технология, Бизнес» за 2019 год, было рассказано об особенностях проектирования различных твердотельных СВч-переключателей средней и большой мощности. В данном номере рассматриваются pin-диодные переключатели нескольких типов, выпускаемые рядом производителей.
\end{abstract}

\section{МОЩНЫЕ РІN-ДИОДНЫЕ ПЕРЕКЛЮЧАТЕЛИ Интегральные переключатели}

Исторически первыми появились модульные переключатели на дискретных ріп-диодах, выполненных по кремниевой технологии. Далее получили развитие монолитные переключатели на кремниевой (табл. 1-3), а затем и на арсенид-галлиевой подложках. Особенно высокие требования по мощности стали предъявляться к переключателям, обеспечивающим подключение передатчика и приемника к одной или нескольким антеннам. Среди кремниевых ріп-диодных переключателей наибольшее применение нашли переключатели "приемпередача» (Rx/Tx) (см. табл. 3), работающие на одну антенну. Для этих применений вполне подходят симметричные SPDT-переключатели (рис. 5a). Однако существенно различные требования к трактам прохождения сигнала от передатчика к антенне и от антенны к приемнику привели к появлению асимметричных SPDT-переключателей с различной схемотехникой каналов (рис. 5б).

Когда передатчик подключается к антенне, сопротивление диода Ді и вносимые в этом канале потери становятся минимальными, а сопротивление диода Д2 в приемном канале становится максимальным, чем обеспечивается защита приемника от большой мощности на выходе передатчика. Дополнительная развязка входа приемника и выхода передатчика достигается переводом диода Дз в открытое состояние. При этом часть просочившейся через диод д2 мощности замыкается на землю. При переходе в приемный режим Д1 и Дз закрываются, а диод д2 переходит в открытое состояние.

Мощный (125 Вт) асимметричный переключатель MASW-000936 (рис. 6), выпускаемый в безвыводном

ООО «Радиокомп», генеральный директор.

МтУси, доцент.

АО «Микро-Вис», заместитель генерального директора по науке. пластиковом PQFN-корпусе, обладает в широкой полосе частот (0,05-6,0 ГГц) высокой (IIP3=72 дБМ) линейностью, и предназначен в первую очередь для WiMAXи WiFi-применений. Переключатель может использоваться как при двухполярном (-15/15 В), так и при однополярном (0/28 В) напряжениях. Необходимые в каждом из этих случаев управляющие напряжения и токи обеспечиваются внешними драйверами.

Серия SPDT-переключателей MSW200x-200 включает три изделия: MSW2000-200 (0,05-1 ГГц), MSW2001-200 (0,4-4 ГГц) и MSW2002-200 (2-6ГГц). Асимметричная структура этих гибридных переключателей (см. рис. 5б) обеспечивает минимум вносимых потерь в канале передатчика и максимальную развязку в канале приемника (рис. 7). Для обеспечения высокой коммутируемой мощности (125 Вт) в переключателях используются высоковольтные ріп-диоды, а все вспомогательные пассивные элементы, обеспечивающие управление переключателями, размещаются на керамической подложке. Чрезвычайно малое тепловое сопротивление ріп-диодов (менее $10{ }^{\circ} \mathrm{C} / \mathrm{BT}$ ) делает их весьма надежными при холодной $\left(\mathrm{T}=85^{\circ} \mathrm{C}\right)$ коммутации сигналов с непрерывной мощностью до 50 дБм и пиковой мощностью до 53 дБм.

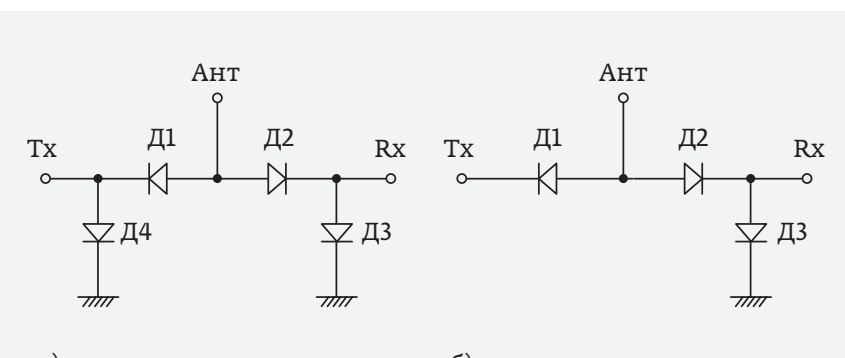

a)

б)

Рис. 5. Типы pin-диодных SPDT-переключателей: a - симметричный; 6 - асимметричный 
Таблица 1. Интегральные переключатели средней мощности на кремниевых ріп-диодах

\begin{tabular}{|c|c|c|c|c|c|c|c|}
\hline Компания & Модель & Тип & $\begin{array}{r}\text { Диапазон } \\
\text { частот, ГГц }\end{array}$ & $\begin{array}{r}\mathrm{P}_{\text {доп }}, \\
\mathrm{BT}\end{array}$ & IL, дБ & Iso, дБ & $\mathrm{T}_{\mathrm{n}}, \mathrm{HC}$ \\
\hline Skyworks Solutions & SKY13525-646LF & SP6T & $0,4-2,7$ & $<4$ & $0,45-0,60$ & $30-20$ & 2000 \\
\hline MACOM & MASW-011052 & SPDT & $2-18$ & 2 & $0,55-1,20$ & $70-48$ & 50 \\
\hline MACOM & MASW-002102-13580 & SPDT & $2-18$ & 2 & $0,7-1,5$ & $60-40$ & 50 \\
\hline MACOM & MASW-003102-13590 & SP3T & $2-18$ & 2 & $0,8-1,6$ & $59-40$ & 50 \\
\hline MACOM & MASW-002103-1363 & SPDT & $0,05-20,0$ & $6,2-2$ & $0,55-1,05$ & $52-27$ & 20 \\
\hline MACOM & MASW-003100-1192 & SP3T & $0,05-20,0$ & 2 & $0,5-0,9$ & $57-42$ & 20 \\
\hline MACOM & MASW-004103-1365 & SP4T & $0,05-20,0$ & $6,2-2$ & $0,5-1,2$ & $51-32$ & 20 \\
\hline
\end{tabular}

Примечание: IL - вносимые потери, Iso - развязка, $\mathrm{T}_{\text {п }}$ - время переключения

Таблица 2. Интегральные переключатели на кремниевых ріп-диодах большой (более 10 Вт) мощности

\begin{tabular}{|c|c|c|c|c|c|c|c|c|}
\hline Компания & Модель & Тип & $\begin{array}{r}\text { Диапазон } \\
\text { частот, ГГц }\end{array}$ & $\begin{array}{c}\mathrm{P}_{\text {доп }}, \\
\mathrm{BT}\end{array}$ & $\begin{array}{r}\mathrm{P}_{\text {и }} / \mathrm{T}_{\text {и }} / \mathrm{Q}, \\
\mathrm{BT} / \mathrm{MKC} / \%\end{array}$ & IL, дБ & Iso, дБ & $T_{n}, M K C$ \\
\hline Microsemi & MPS2R10-606 & SPDT & $0,05-1,0$ & 100 & - & $0,1-0,5$ & $60-47$ & - \\
\hline Microsemi & MPS4103-607 & SPST & $0,1-3,0$ & 40 & - & $0,1-0,5$ & $70-44$ & 0,5 \\
\hline MACOM & MASW-011040 & SP4T & $0,05-1,0$ & $200-80$ & - & $0,25-0,45$ & $51-45$ & 3,5 \\
\hline MACOM & MSW2060-206 & SPDT & $0,02-1,0$ & 100 & $500 / 10 / 1$ & 0,25 & 53 & 2 \\
\hline MACOM & MSW2061-206 & SPDT & $0,4-4,0$ & 100 & $500 / 10 / 1$ & 0,5 & 35 & 1 \\
\hline MACOM & MSW3201-320 & SP3T & $0,4-4,0$ & 100 & $500 / 10 / 1$ & 0,6 & 35 & 1,0 \\
\hline MACOM & MSW2032-203 & SPDT & $2,0-6,0$ & 100 & $500 / 10 / 1$ & 0,6 & 35 & 1,0 \\
\hline MACOM & MSW2062-206 & SPDT & $2,0-6,0$ & 100 & $500 / 10 / 1$ & 0,7 & 34 & 1 \\
\hline MACOM & MASW-011060 & SPDT & $0,5-6,0$ & 158 & - & $0,15-0,80$ & $47-28$ & 1,5 \\
\hline MACOM & MASW-011071 & SPDT & $8,0-10,5$ & 20 & - & $0,9-1,2$ & $36-39$ & 0,13 \\
\hline KCB Solutions & KS113-52 & SPDT & $0,02-2,0$ & 50 & - & $0,03-0,75$ & 45 & - \\
\hline KCB Solutions & KS03R2-22 & SPDT & $0,02-3,0$ & 200 & - & $0,4-0,7$ & $30-22$ & - \\
\hline KCB Solutions & KS03R3-22 & SP3T & $0,02-3,0$ & 200 & - & $0,4-0,7$ & $30-22$ & - \\
\hline KCB Solutions & KS102-55 & SPDT & $0,225-3,5$ & 100 & - & $0,25-1,20$ & $45-23$ & 0,05 \\
\hline Skyworks Solutions & SKY12211-478LF & SPDT & $0,05-2,7$ & 60 & $240 / 10 / 4$ & $0,22-0,53$ & $50-32$ & 0,032 \\
\hline Skyworks Solutions & SKY12213-478LF & SPST & $0,5-6,0$ & 190 & $760 / 10 / 4$ & $0,27-1,30$ & $34-20$ & 0,19 \\
\hline Wei Bo Associates & MSW2T-2040X-194 & SPDT & $0,1-1,0$ & 320 & $1000 / 10 / 1$ & 0,15 & 45 & 40 \\
\hline Wei Bo Associates & MSW2T-2041-193 & SPDT & $0,4-4,0$ & 160 & $500 / 10 / 1$ & 0,5 & 33 & 2,0 \\
\hline Wei Bo Associates & MSW2T-2060-195 & SPDT & $0,02-1,2$ & 100 & $500 / 10 / 1$ & 0,25 & 53 & 0,75 \\
\hline Wei Bo Associates & MSW2T-2061-195 & SPDT & $0,2-4,5$ & 100 & $500 / 10 / 1$ & 0,5 & 35 & 0,75 \\
\hline Wei Bo Associates & MSW2T-2062-195 & SPDT & $1,5-6,5$ & 100 & $500 / 10 / 1$ & 0,7 & 34 & 0,75 \\
\hline Wei Bo Associates & MSW2T-8512-740 & SPDT & $8,5-12,0$ & 100 & $400 / 10 / 1$ & 0,7 & 35 & 1,1 \\
\hline
\end{tabular}


Таблица 3. Интегральные переключатели “прием-передача» на кремниевых ріп-диодах

\begin{tabular}{|c|c|c|c|c|c|c|c|}
\hline Компания & Модель & $\begin{array}{r}\text { Диапазон } \\
\text { частот, } \\
\text { ГГц }\end{array}$ & $\begin{array}{r}\mathrm{P}_{\text {доп }} \\
(\mathrm{T} x / \mathrm{Rx}) \\
\mathrm{BT}\end{array}$ & $\begin{array}{r}P_{\text {и }} / \mathrm{T}_{\text {и }} / \mathrm{Q}, \\
\mathrm{BT} / \mathrm{MKC} / \%\end{array}$ & $\begin{array}{r}\mathrm{IL}(T x / R x), \\
\text { дБ }\end{array}$ & $\begin{array}{r}\text { Iso }(T x / R x), \\
\text { дБ }\end{array}$ & $\begin{array}{r}T_{\Pi}, \\
M K C\end{array}$ \\
\hline MACOM & MSW2022-202 & $0,05-1,0$ & $160 / 10$ & $500 / 10 / 1$ & $0,2 / 0,3$ & $45 / 23$ & 1,5 \\
\hline Wei Bo Associates & MSW2T-2050-194 & $0,05-1,0$ & $160 / 20$ & $640 / 10 / 1$ & $0,15 / 0,25$ & $50 / 26$ & 1,0 \\
\hline Wei Bo Associates & MSW2T-0025-195 & $1,0-2,0$ & $100 / 10$ & $500 / 10 / 1$ & $0,3 / 0,3$ & $20 / 15$ & 1,5 \\
\hline Skyworks Solutions & SKY12212-478LF & $0,05-2,7$ & $120 / 60$ & $480 / 10 / 4$ & $\begin{array}{r}(0,29-0,55) / \\
(0,24-0,44)\end{array}$ & $\begin{array}{l}(59-28) / \\
(47-29)\end{array}$ & 0,6 \\
\hline MACOM & MSW-2002-200 & $2,0-6,0$ & $100 / 20$ & $500 / 10 / 1$ & $0,6 / 0,9$ & $34 / 13$ & 1,0 \\
\hline Skyworks Solutions & SKY12210-478LF & $0,9-4,0$ & $120 / 60$ & $480 / 10 / 4$ & $\begin{array}{r}(0,33-0,52) / \\
(0,26-0,56)\end{array}$ & $\begin{array}{r}(35-25) / \\
(44-33)\end{array}$ & 0,157 \\
\hline MACOM & MSW2011-201 & $0,4-4,0$ & $100 / 20$ & $500 / 10 / 1$ & $0,3 / 0,4$ & $36 / 14$ & 1,5 \\
\hline Wei Bo Associates & MSW2T-2051-194 & $0,4-4,0$ & $160 / 120$ & $640 / 10 / 1$ & $0,3 / 0,8$ & $34 / 13$ & 1,0 \\
\hline MACOM & MASW-000936 & $0,05-6,0$ & $\begin{array}{r}(120-80) / \\
14\end{array}$ & $1000 / 10 /-$ & $\begin{array}{r}(0,07-0,25) / \\
(0,20-0,70)\end{array}$ & $\begin{array}{r}(16-11) / \\
(50-40)\end{array}$ & 0,2 \\
\hline MACOM & MASW-000825-12770T & $0,05-6,0$ & $20 / 8$ & $200 / 5 / 1$ & $\begin{array}{r}(0,29-0,59) / \\
(0,42-0,95)\end{array}$ & $\begin{array}{r}(24,2-18,5) / \\
(28,6-22,4)\end{array}$ & 0,2 \\
\hline MACOM & MASW-000932 & $0,01-6,0$ & $80 / 14$ & $2000 / 5 / 1$ & $\begin{array}{r}(0,25-0,45) / \\
(0,60-0,80)\end{array}$ & $\begin{array}{r}(14-10) / \\
(43-35)\end{array}$ & 0,2 \\
\hline
\end{tabular}

Малое сопротивление диодов в открытом состоянии обеспечивает высокую линейность (IIP3 > 65 дБм) этих переключателей. Предназначенные для поверхностного монтажа, эти высоконадежные изделия используются в коммерческих, промышленных и военных применениях.

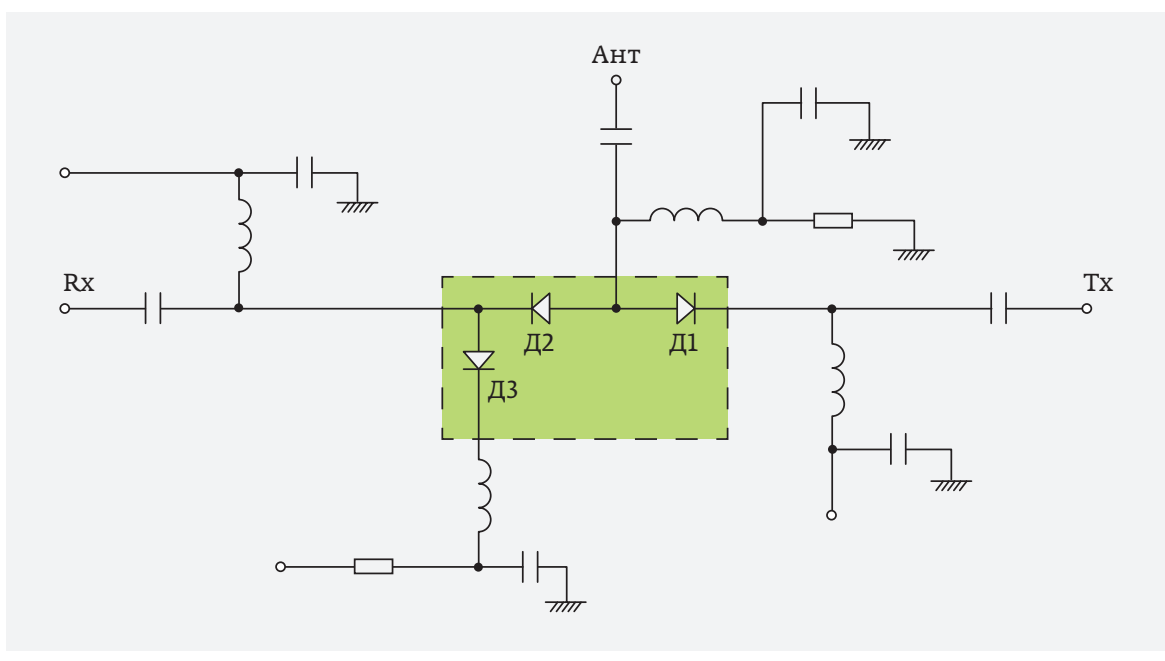

Рис. 6. Принципиальная схема асимметричного ріп-диодного переключателя MASW-000936 (выделен цветом) компании MACOM
Многие недостатки, свойственные кремниевым ріпдиодным переключателям, преодолены в переключателях, изготовленных по НМІС-технологии, выпускаемых компанией МАСОМ. В рамках этой технологии кремниевые вставки с диодами размещены на поверхности стеклянной подложки, используемой для формирования на ней пассивных элементов, обеспечивающих управление ріп-диодами (рис. 8а). Найденное техническое решение позволило сформировать на стекле топологию (рис. 8б) с очень высоким разрешением, обеспечивающим высокую повторяемость характеристик в том числе и на высоких частотах.

Асимметричный SPDT-переключатель (см. рис. 5б) был реализован в монолитном исполнении [15] с использованием НMIC-технологии. В этом техническом решении применяются только положительные источники питания, что достигается развязкой по постоянному току катода шунтирующего диода в приемном канале. Этот асимметричный 
Таблица 4. Арсенид-галлиевые ріп-диодные переключатели средней мощности в интегральном исполнении

\begin{tabular}{|c|c|c|c|c|c|c|c|}
\hline Компания & Модель & Тип & $\begin{array}{r}\text { Диапазон } \\
\text { частот, ГГц }\end{array}$ & $P_{\text {доn }}$, ДБМ & IL, дБ & Iso, дБ & $\mathrm{T}_{\Pi}, \mathrm{HC}$ \\
\hline Qorvo & TGS2304-SCC & SP4T & $0,2-20$ & 33 & $0,4-1,3$ & $32-44$ & - \\
\hline Analog Devices & HMC970 & SPDT & $8-21$ & 34 & $0,9-1,4$ & 40 & - \\
\hline Bowei & BW142 & SPST & $4-26$ & 30 & 0,4 & 22 & - \\
\hline Bowei & BW143 & SPST & $0,1-30$ & 30 & 0,5 & 18 & - \\
\hline Qorvo & TGS4304 & SPDT & $32-40$ & 35 & $0,9-1,3$ & - & 4 \\
\hline MACOM & MASW-011036 & SPDT & $26-40$ & 43 & $0,7-1,5$ & $38-40$ & $<30$ \\
\hline Alpha & AP640R5-00 & SPDT & $24-40$ & 40 & $0,8-1,8$ & 34 & 2 \\
\hline Alpha & AP640R1-00 & SPST & $18-40$ & 40 & $1,0-1,3$ & $42-36$ & 4 \\
\hline Analog Devices & HMC971 & SPDT & $18-40$ & 35 & $1,0-1,5$ & 40 & - \\
\hline Qorvo & TGS4302 & SPDT & $27-46$ & 37 & $0,9-1,3$ & - & 4 \\
\hline Analog Devices & HMC975 & SPDT & $2-50$ & $23-30$ & $0,5-1,7$ & $50-45$ & - \\
\hline Qorvo & TCS4306-FC & SP4T & $70-90$ & 27 & 2,5 & 20 & $<5$ \\
\hline Qorvo & TGS4305-FC & SP3T & $60-90$ & 27 & 1,6 & 20 & $<5$ \\
\hline
\end{tabular}

переключатель, разработанный компанией МАСОМ, обеспечивает достаточно большую коммутируемую мощность $\left(P_{\text {н }}=40\right.$ Вт, $P_{\text {и }}=1000$ Вт), а также низкие вносимые потери и хорошую развязку. Полученные результаты достигнуты благодаря эффективным решениям, выработанным на основе электромагнитных и тепловых расчетов, включая выбор прямоугольной формы диода Д1 в передающем канале (рис. 9).

Наряду с традиционными зависимостями IL(f), Iso(f) и др., имеющимися в технических описаниях (data sheets), для мощных переключателей приводятся и другие данные, позволяющие определиться с выбором самого

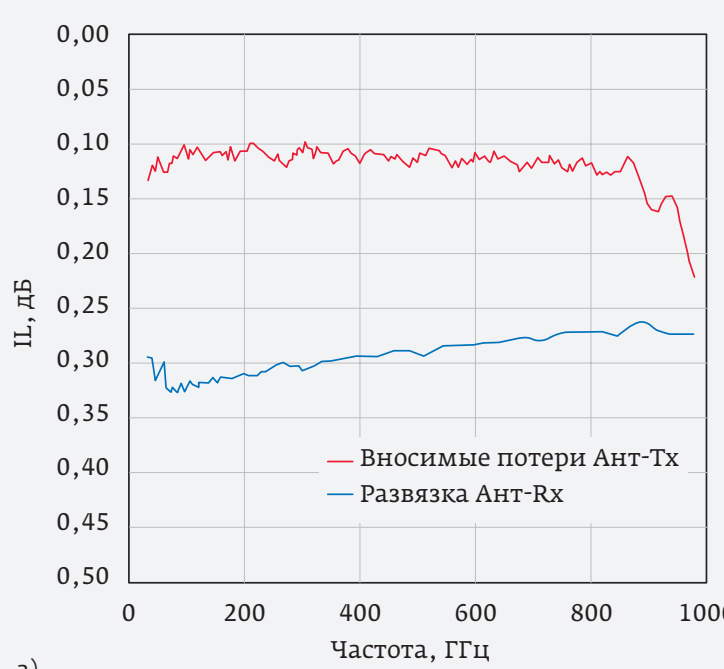

a)

0,00
10,00
20,00
30,00
40,00
50,00
60,00
70,00
80,00
90,00
100,00
0

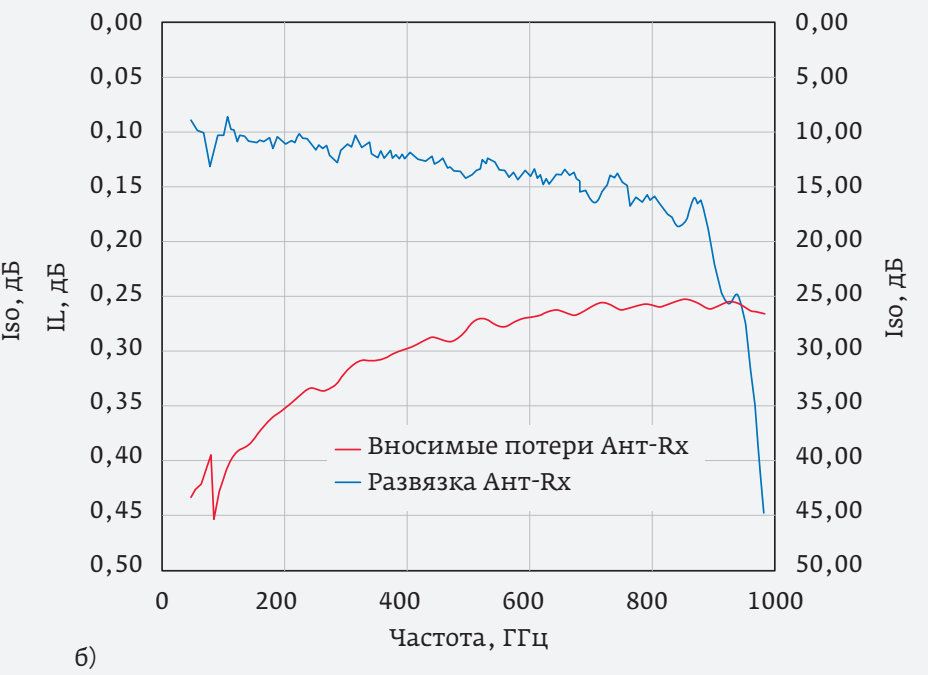

Рис. 7. Зависимости вносимых потерь IL и развязки Iso от частоты в асимметричном ріп-диодном переключателе MSW2000-200 в передающем (а) и приемном (б) каналах 
Таблица 5. Мощные SPST-, SPDT-, DPDT-переключатели на кремниевых ріп-диодах в модульном исполнении

\begin{tabular}{|c|c|c|c|c|c|c|c|c|}
\hline Компания & Модель & Тип & $\begin{array}{r}\text { Диапазон } \\
\text { частот, ГГц }\end{array}$ & $\begin{array}{c}\mathrm{P}_{\text {доп }}, \\
\mathrm{BT}\end{array}$ & $\begin{array}{r}\mathrm{P}_{\text {и }} / \mathrm{T}_{\text {и }} / \mathrm{Q}, \\
\mathrm{BT} / \mathrm{MKC} / \%\end{array}$ & $\begin{array}{l}\text { IL, } \\
\text { дБ }\end{array}$ & $\begin{array}{l}\text { Iso, } \\
\text { дБ }\end{array}$ & $\begin{array}{r}\mathrm{T}_{\mathrm{n}}, \\
\mathrm{MKC}\end{array}$ \\
\hline Temex & SH90207 & SPDT & $0,0015-0,05$ & 1000 & - & $<0,15$ & $>37$ & - \\
\hline Aethercomm & SSHPS $0.225-0.450-400$ & SPDT & $0,225-0,450$ & 400 & $2000 /-/-1$ & 0,7 & 40 & 5 \\
\hline $\begin{array}{l}\text { Seekon } \\
\text { Microwave }\end{array}$ & XK29655054 & SPDT & $0,1-0,5$ & 150 & - & $<0,8$ & $>30$ & 0,9 \\
\hline Cobham & SH91107 & SPDT & $0,02-0,50$ & 1000 & - & $<0,2$ & $>33$ & - \\
\hline Elisra & MWP-41211-CEL & SPST & $0,824-0,960$ & 200 & $2000 /-1-$ & $0,4-0,6$ & $35-30$ & - \\
\hline $\begin{array}{l}\text { Mercury } \\
\text { Systems }\end{array}$ & ES0232 & SPDT & $0,5-1,0$ & 1000 & - & $<0,5$ & $>45$ & 40 \\
\hline CPI & MA8305-2L18SD & SPDT & $1,02-1,10$ & - & $500 /-/-$ & $<0,7$ & $>40$ & $<120$ \\
\hline LabTech & LMS902 & SPDT & $1,0-1,1$ & - & $<500 /-/-$ & $<0,8$ & $>45$ & 1 \\
\hline Aethercomm & SSHPS 1.2-1.4-4000 & SPDT & $1,2-1,4$ & $<400\left(\mathrm{P}_{\mathrm{cp}}\right)$ & $4000 / 80 / 10$ & $0,23-0,30$ & 53,7 & 1,8 \\
\hline PMI & $\begin{array}{l}\text { P2T-1200M1400M- } \\
\text { 35-R-SFF }\end{array}$ & SPDT & $1,2-1,4$ & - & $500 / 10 / 1$ & $<0,4$ & $>35$ & 5 \\
\hline Kratos & SW-2367-01 & SPDT & $1,4-1,8$ & $<60$ & - & $<1,0$ & $>35$ & 10 \\
\hline RF-Lambda & RFSP2TR0003GK & SPDT & $0,02-2,55$ & 150 & $1000 / 1 / 5$ & $1,2-2,0$ & 50 & 5 \\
\hline KORTCOM & KWM05080A & DPDT & $2,5-2,7$ & $200,50\left(P_{\mathrm{cp}}\right)$ & $600 /-/-$ & $<1,0$ & $>100$ & 3 \\
\hline Aethercomm & SSHPS 2.7-3.5-1000 & SPDT & $2,7-3,5$ & 50 & $1000 /-/-$ & 0,9 & 40 & 4 \\
\hline CPI & VLS1522A & SPDT & $3,05-3,55$ & 50 & $1000 / 100 / 5$ & $<1,0$ & $>25$ & 5 \\
\hline Радиокомп & PKK-4-500 & SPDT & $0,5-4,0$ & 160 & $500 /-/-$ & 1,0 & 70 & 5 \\
\hline Linwave & LW24-793219 & SPDT & $4,1-4,7$ & 25 & $200 / 250 / 10$ & 1,7 & - & 6 \\
\hline CPI & VLC1599 & SPST & $5,25-5,75$ & - & $2000 / 640 / 20$ & $<1,2$ & $>30$ & 10 \\
\hline Aeroflex & ACSW-5734 & $\begin{array}{l}\text { SPDT } \\
(\mathrm{Tx} / \mathrm{Rx})\end{array}$ & $2,0-6,0$ & 100 & - & $\begin{array}{l}<1,5(\mathrm{Rx}) \\
<0,5(\mathrm{Tx})\end{array}$ & $\begin{array}{r}<70-50 \\
(\mathrm{Rx}) \\
<15(\mathrm{Tx})\end{array}$ & 75 \\
\hline $\begin{array}{l}\text { Mercury } \\
\text { Systems }\end{array}$ & ES0008 & SPDT & $4,0-8,0$ & $80\left(P_{c p}\right)$ & $2000 / 10 / 4$ & - & - & 2 \\
\hline $\begin{array}{l}\text { Exodus } \\
\text { Advanced } \\
\text { Communica- } \\
\text { tions }\end{array}$ & PIN1013 & SPDT & $2,0-8,0$ & - & $2000 / 10 / 5$ & $<1,5$ & $>40$ & 0,15 \\
\hline $\begin{array}{l}\text { RH } \\
\text { Laboratories }\end{array}$ & $120-F 213$ & SPDT & $8,0-12,4$ & $210\left(\mathrm{P}_{\mathrm{cp}}\right)$ & $3500 / 10 /-$ & 1,6 & $>40$ & 1,8 \\
\hline Weinschel & 8501 & SPDT & $0-13,0$ & $\begin{array}{c}100 \text { (при } \\
\text { горячем } \\
\text { переклю- } \\
\text { чении) }\end{array}$ & - & $0,25-0,65$ & $70-50$ & - \\
\hline
\end{tabular}


Таблица 5. Продолжение

\begin{tabular}{|c|c|c|c|c|c|c|c|c|}
\hline Компания & Модель & Тип & $\begin{array}{r}\text { Диапазон } \\
\text { частот, ГГц }\end{array}$ & $\begin{array}{c}\mathrm{P}_{\text {доп }}, \\
\mathrm{BT}\end{array}$ & $\begin{array}{r}P_{\text {и }} / \mathrm{T}_{\text {и }} / \mathrm{Q}, \\
\mathrm{BT} / \mathrm{MKc} / \%\end{array}$ & $\begin{array}{l}\text { IL, } \\
\text { дБ }\end{array}$ & $\begin{array}{l}\text { Iso, } \\
\text { дБ }\end{array}$ & $\begin{array}{r}\mathrm{T}_{\text {п }}, \\
\mathrm{MKC}\end{array}$ \\
\hline $\begin{array}{l}\text { RH } \\
\text { Laboratories }\end{array}$ & $120-F 219$ & SPDT & $12,4-18,0$ & $120\left(\mathrm{P}_{\mathrm{cp}}\right)$ & $2000 / 10 /-$ & $<1,8$ & $>40$ & 1,8 \\
\hline Comtech & H26-206 & SPDT & $6,0-18,0$ & 80 & $2000 / 5 / 4$ & 2,5 & $>40$ & 1,5 \\
\hline A-INFO & KG-1R-60180-120 & SPST & $6,0-18,0$ & 120 & - & $<1,4$ & $>20$ & 0,8 \\
\hline $\begin{array}{l}\text { Seekon } \\
\text { Microwave }\end{array}$ & XK296530029 & SPST & $6,0-18,0$ & 120 & - & $<1,4$ & $>20$ & 0,5 \\
\hline HXI & HSWM22801-309 & SPDT & $26,5-40,0$ & 10 & - & 1,3 & 38 & 0,03 \\
\hline
\end{tabular}

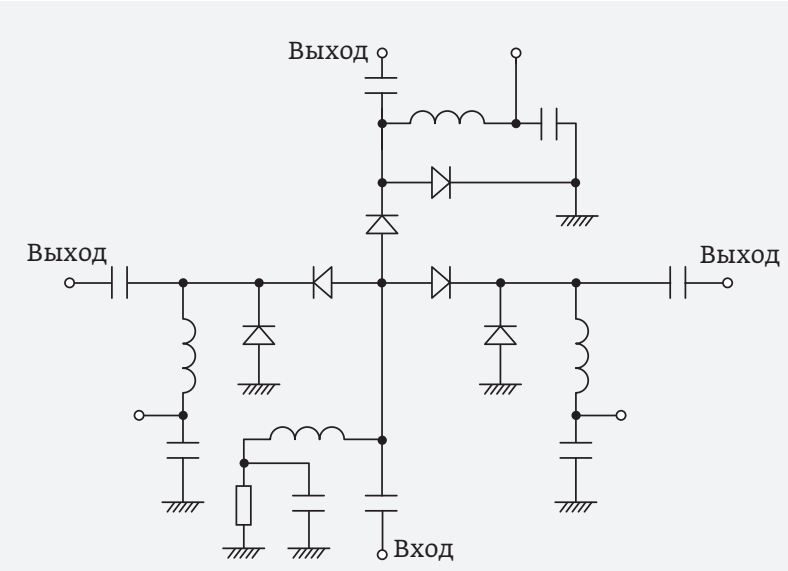

a)

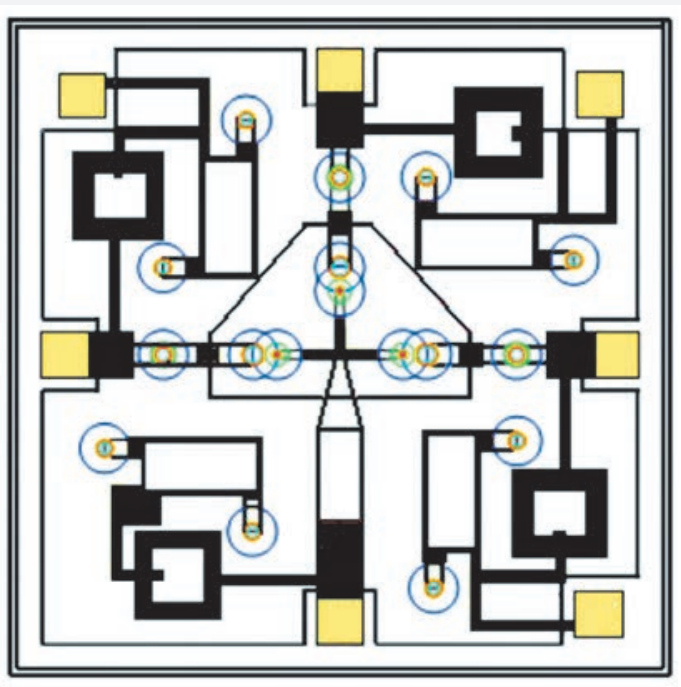

б)

Рис. 8. Принципиальная схема (а) и топология (б) pinдиодного SP3Т-переключателя MASW-003102-13590 (компания МАСОМ) переключателя, с пропускаемой через него мощностью, управляющими напряжениями и токами, исходя из существующих ограничений.

Использование компанией МАСОМ технологии НMIC позволило разработать ей достаточно широкую линейку переключателей SPDT-, SP3T-и SP4T-типов. При этом в каждом из каналов использовались все три переключающих структуры: последовательная, параллельная и последовательно-параллельная, а также асимметричная структура, включающая последовательный тип переключателя в передающем канале и последовательнопараллельный тип в приемном канале.

Максимальная коммутируемая мощность 200 Вт была достигнута компанией MACOM в SP4T-переключателе MASW-011040 в диапазоне частот 0,05-1,0 ГГц. Коммутируемые мощности 100-120 Вт удалось получить на частотах до бГГц (MSWSH-100-30, MASW000936),

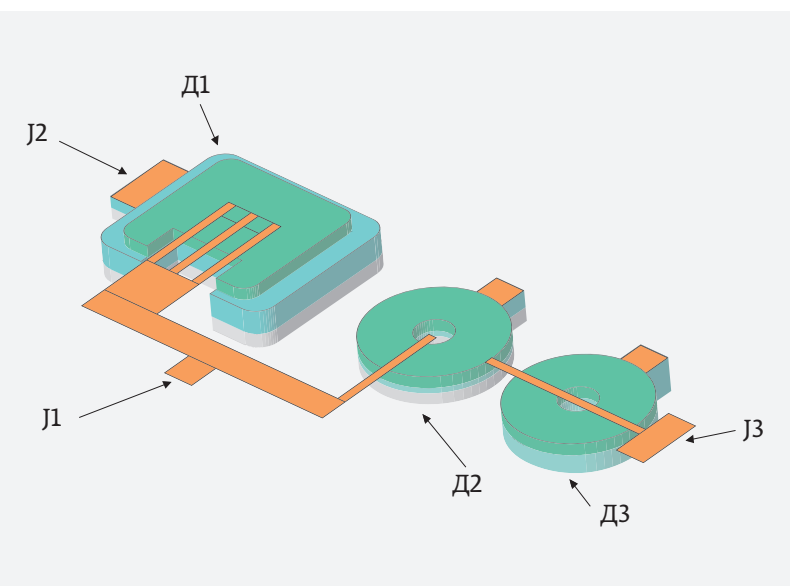

Рис. 9. Упрощенная конструкция ріп-диодного переключателя большой (1000 Вт) мощности, реализованного по технологии НМІС (компания МАСОМ). J1 - вход, J2, Ј3 -выходы 
Таблица 6. Многопозиционные (SP3T, SP4T, ..., SP160T) переключатели на ріп-диодах в модульном исполнении

\begin{tabular}{|c|c|c|c|c|c|c|c|}
\hline Компания & Модель & Тип & $\begin{array}{r}\text { Диапазон } \\
\text { частот, } \\
\text { ГГц }\end{array}$ & $\begin{array}{c}\mathrm{P}_{\text {доп }}, \\
\mathrm{BT}\end{array}$ & $\begin{array}{l}\text { IL, } \\
\text { дБ }\end{array}$ & $\begin{array}{l}\text { Iso, } \\
\text { дБ }\end{array}$ & $\begin{array}{r}T_{n}, \\
M K C\end{array}$ \\
\hline Temex & SH92103 & SP3T & $0,02-1,0$ & 100 & $<0,15$ & $>37$ & - \\
\hline AA-MCS & $\begin{array}{l}\text { AAMCS-SWT-SP3T- } \\
400 M-4000 M-50 \mathrm{dBm}\end{array}$ & SP3T & $0,4-4,0$ & 100 & 1,0 & 30 & 5 \\
\hline $\begin{array}{l}\text { Mercury } \\
\text { Systems }\end{array}$ & SA5NA4 & SP4T & $0,5-18,0$ & $<0,5$ & $<3,4$ & $>60$ & 0,1 \\
\hline Omicron & JXSP4TA0020C & SP4T & $0,8-20,0$ & 2 & 3 & 70 & 0,05 \\
\hline $\begin{array}{l}\text { SACE } \\
\text { Millimeter }\end{array}$ & SK4-1835035030-2F2F-R1 & SP4T & $18,0-50,0$ & 0,25 & 5 & 30 & 0,1 \\
\hline ETL & SW-SP7T-C1-1605 & SP7T & $3,0-5,0$ & 1 & 2,8 & $>80$ & 0,1 \\
\hline CMC & SR70-313 & SP7T & $0,1-20,0$ & 2 & $1,1-4,6$ & $75-60$ & 1 \\
\hline Elisra & MWP-41218-PCS & SP8T & $1,85-2,15$ & $\begin{array}{r}200 \\
P_{\text {и }}=2000\end{array}$ & $1,0-1,2$ & $30-25$ & - \\
\hline UMCC & $\mathrm{SR}-\mathrm{C} 00 \mathrm{H}-8 \mathrm{~S}$ & SP8T & $0,5-2,7$ & $<2$ & $<1,3$ & $>40$ & 0,1 \\
\hline $\begin{array}{l}\text { Corry } \\
\text { Micronics }\end{array}$ & CMIK8-214-111 & SP8T & $2,0-18,0$ & $<1$ & 3,7 & $>80$ & 0,05 \\
\hline Omicron & JXSP8TA0118C & SP8T & $1,0-18,0$ & 2 & 4 & 80 & 0,05 \\
\hline RF-Lambda & RFSP8T0220C & SP8T & $2,0-20,0$ & $0,5-5,0\left(P_{\mathrm{cp}}\right)$ & 2,5 & $>80$ & 0,1 \\
\hline Cernex & C10TA08185070 & SP10T & $8,0-18,0$ & 0,2 & 5,0 & $>70$ & 0,05 \\
\hline AMC & MSN-10DR/DT-05-STD & SP10T & $0,5-18$ & 0,1 & $<5,0$ & $>60$ & 0,1 \\
\hline Kratos & 1744 & SP16T & $2,0-18,0$ & 1 & $<6,0$ & $60-50$ & 0,5 \\
\hline RF-Lambda & RFSP16TA5M43C & SP16T & $0,5-43,5$ & 1 & 14 & 60 & 0,07 \\
\hline G.T.Microwave & S9L-51-0BX & SP17T & $4,5-5,0$ & 3 & 3,0 & 30 & 1 \\
\hline RF-Lambda & RFSP32TA5M43C & SP32T & $0,5-43,5$ & $<1$ & 28 & 60 & 0,07 \\
\hline AMC & MSN-48DT-05-DEC-MP & $\mathrm{SP} 48 \mathrm{~T}$ & $18,0-21,5$ & 0,1 & $<11,5$ & $>80$ & 0,25 \\
\hline AMC & MSN-65DT-051-DEC-MP & SP65T & $0,1-4,0$ & 0,1 & $<15$ & $>40$ & 0,15 \\
\hline RF-Lambda & RFSP160TA0020C & SP160T & $0,05-20,0$ & $<1$ & 28 & 60 & 0,07 \\
\hline
\end{tabular}

20 Вт - в диапазоне 8,0-10,5 ГГц (MASW-011071) и 2 Вт на частоте 20 ГГц (MASW-001100-1191, MASW-001100-192, MASW-003103-1364, MASW-004103-1365).

Ряд новых изделий компании МАСОМ был скопирован гонконгской компанией Wei Bo Associates и выпускается в настоящее время под теми же названиями. Несколько моделей гонконгских переключателей представлено в табл. 2 и 3. По данным технических описаний их характеристики в некоторых моделях превосходят характеристики оригинальных изделий, разработанных MACOM.
Значительный вклад в разработку мощных СВЧ-переключателей на кремниевых ріп-диодах внесла компания Skyworks Solutions (см. табл. 2, 3), выпустившая на рынок 120-Вт изделия SKY12210-478LF, рассчитанные на диапазон частот 0,9-4,0 ГГц, и 190-Вт переключатели SKYI2213-478LF, работающие в диапазоне 0,5-6,0 ГГц.

Много полезных сведений, помогающих разработчику определиться с возможностью применения переключателей в различных условиях, содержится в технических описаниях. Наглядным примером здесь могут служить зависимости $\operatorname{IL}\left(\mathrm{P}_{\mathrm{BX}}\right)$ и Iso $\left(\mathrm{P}_{\mathrm{BX}}\right)$ при различных 

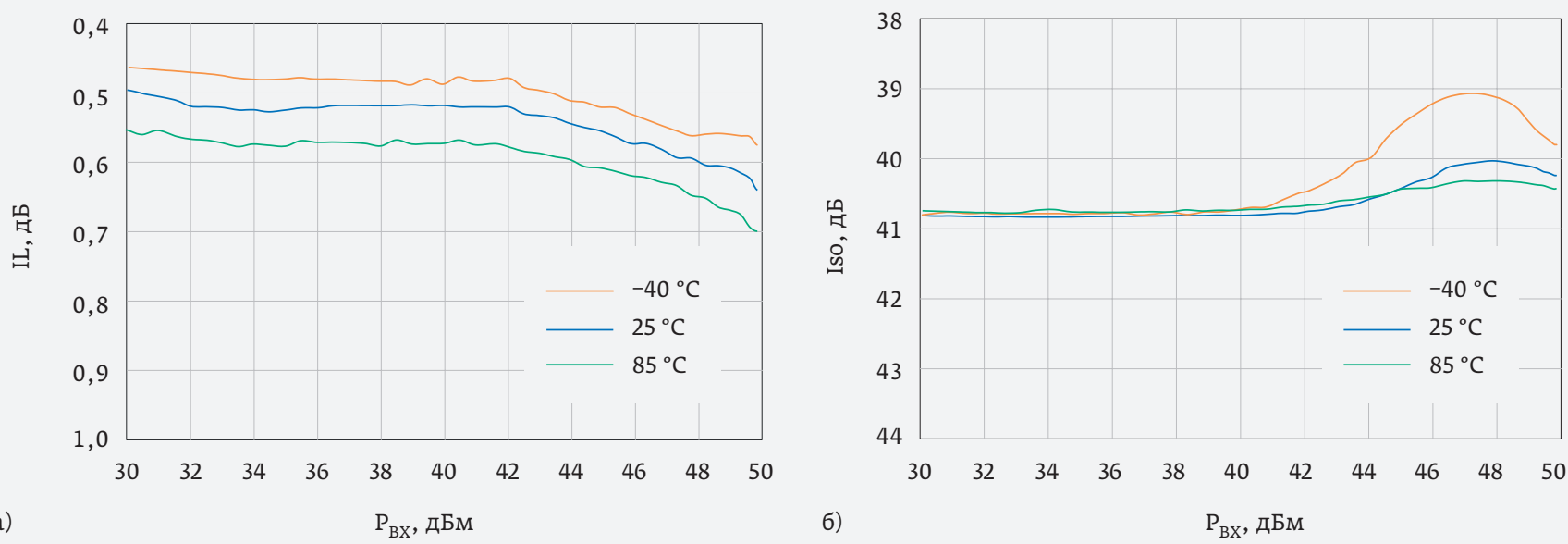

Рис. 10. Зависимости вносимых потерь (а) и развязки (б) от уровня входной мощности для нескольких значений температуры (модель SKY12210-478L8 компании Skyworks Solutions)

температурах окружающей среды (рис. 10) и различных напряжениях смещения (рис. 11), а также зависимости максимальной входной мощности $\mathrm{P}_{\text {вх макс }}$ от температуры печатной платы (рис. 12), с установленным на ней переключателем MASW-000825-12770T.

В начале статьи было отмечено, что температура p-n-перехода последовательно включенного в передающий тракт переключателя не должна превышать 150 C. Зависимость этой температуры от уровня входной мощности и пропускаемого через диод тока иллюстрируется графиками на рис. 13. Практический интерес представляют также зависимости, показывающие снижение максимально допустимой входной мощности от ее номинального значения (при температуре $25^{\circ} \mathrm{C}$ ) с увеличением температуры на нижней поверхности корпуса переключателя (рис. 14а) и на нижней поверхности печатной платы, на которую установлен переключатель (рис. 146).

Из новых разработок мощных интегральных ріп-диодных переключателей стоит отметить продукцию компании KCB Solutions, предлагающей широкую линейку
SPST...SP7T стандартных и заказных переключателей. Изделия компании с коммутируемой мощностью 50-200 Вт на частотах до 3 ГГц выпускаются как в стандартных QFN-корпусах (рис. 15а) при коммутируемых мощностях менее 100 Вт, так и во фланцевом исполнении (рис. 15б) при $\mathrm{P}_{\text {доп }}=200$ Вт. Эти переключатели идеально подходят для портативных средств военной связи, проходят 100\%-ное тестирование и эффективно отводят выделяемое тепло. Коммутация 200-Вт мощностей обеспечивается при больших управляющих напряжениях $-500 \ldots 0,7$ В (KS03R2-22, KS03R3-22). Мощности до 50 Вт в переключателе KS113-52 коммутируются управляющими напряжениями \pm 250 В.

В состав переключателей, производимых компанией KCB Solutions, входят блокировочные емкости. Полная схема SPDT-переключателя KS113-52 включает также и внешние элементы (рис. 16). Номер подключаемого канала в этой схеме определяется значениями управляющих напряжений. О высоких характеристиках СВЧ-переключателей, производимых компанией КСB Solutions, свидетельствуют табличные данные (см. табл. 2).

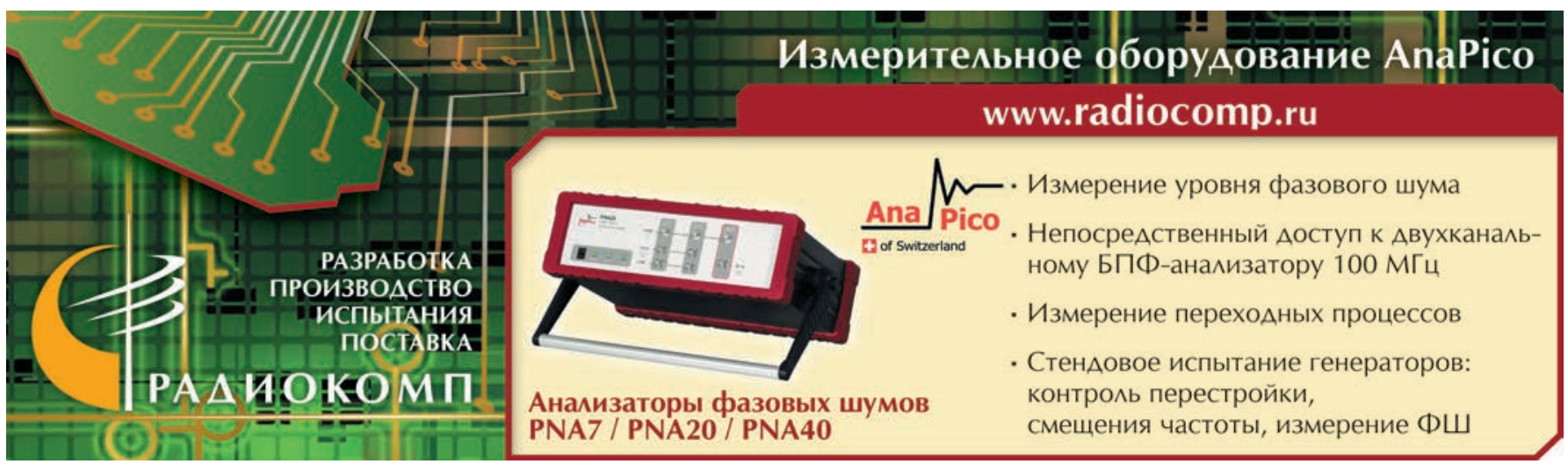



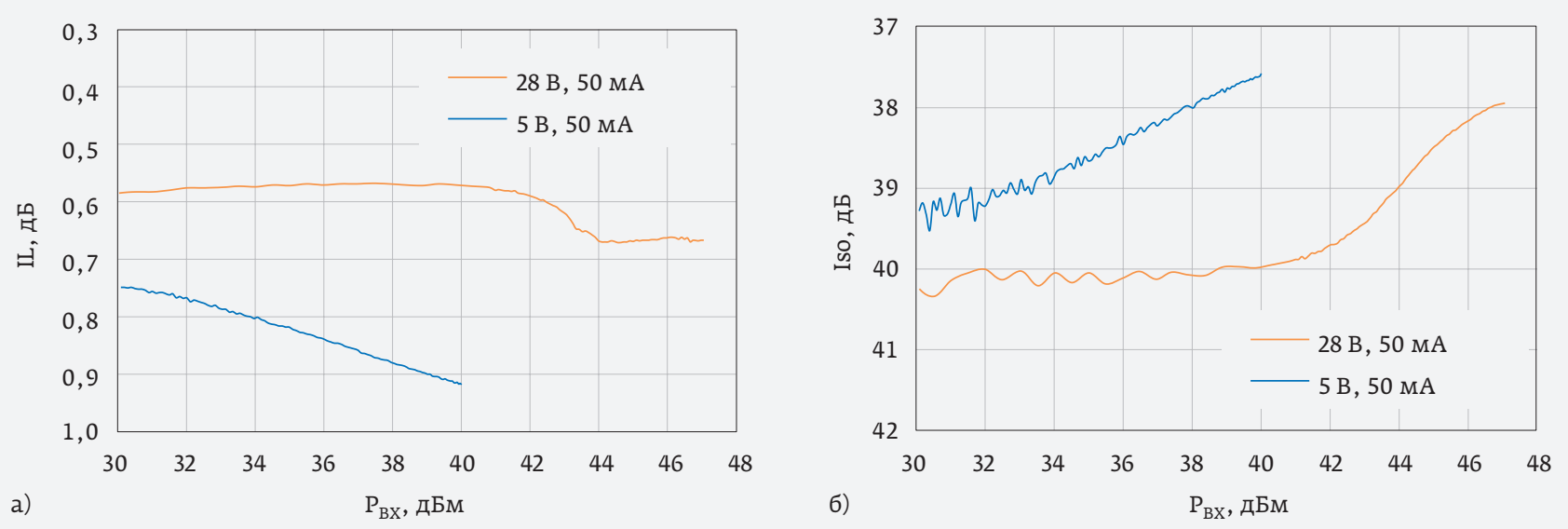

Рис. 11. Зависимости вносимых потерь (а) и развязки (б) от уровня входной мощности при различных напряжениях смещения (модель SKY12211-478LF, компания Skyworks Solutions)

Недавно появившийся на рынке переключатель KS102-55 в QFN-корпусе работоспособен при входных мощностях до 100 Вт. В отличие от переключателя KS113-52 в этом изделии управляющее напряжение меняется в пределах от 0 до 250 В. Рабочий диапазон переключателя лежит в пределах 0,25-3,5 ГГц. Принципиальная схема переключателя незначительно отличается от представленной на рис. 16.

При создании своих SPST...SP7T переключателей кOMпания KCB Solutions применяет последовательный и последовательно-параллельный типы переключателей, используя при создании приемо-передающих переключателей асимметричные решения. Например, в SP3T Tx/ Rx переключателе асимметричное решение используется для подключения антенны к передатчику Тх и двум приемникам Rx1 и Rх2 (рис. 17).

Кремниевые ріп-диодные переключатели отличаются высокой коммутируемой мощностью, но весьма сложны в изготовлении, требуют внешних драйверов и плохо совместимы с другими технологиями. По некоторым характеристикам от кремниевых ріп-диодных переключателей выгодно отличаются интегральные переключатели на арсенид-галлиевой подложке, позволяющие размещать на ней не только ріп-диоды, но и другие компоненты: резисторы, конденсаторы, индуктивности. Эти переключатели (TGS4305-FC, TCS4306-FC), выполненные по технологии GaAs VPIN (vertical PIN), позволяют коммутировать мощности до 0,5 Вт, обладают высокими (до 90 ГГц) рабочими частотами и чрезвычайно малыми временами переключения, которые не превосходят нескольких наносекунд (табл. 4), а также приемлемыми значениями тока управления (примерно 10 мА на канал). Компания МАCOM, в которой получила развитие AlGaAs HMIC технология, обеспечила в изделии MASW-011036 переключение 20-Вт входной мощности в диапазоне 26-40 ГГц.

Принципиальная схема одного из GaAs VPIN переключателей с интегрированными драйверами, производимого компанией Qorvo, и топология интегральной схемы приведены на рис. 18а, б соответственно.

В научной литературе приводятся также сведения о ріп-диодных переключателях, реализованных по нитрид-галлиевой технологии [16].

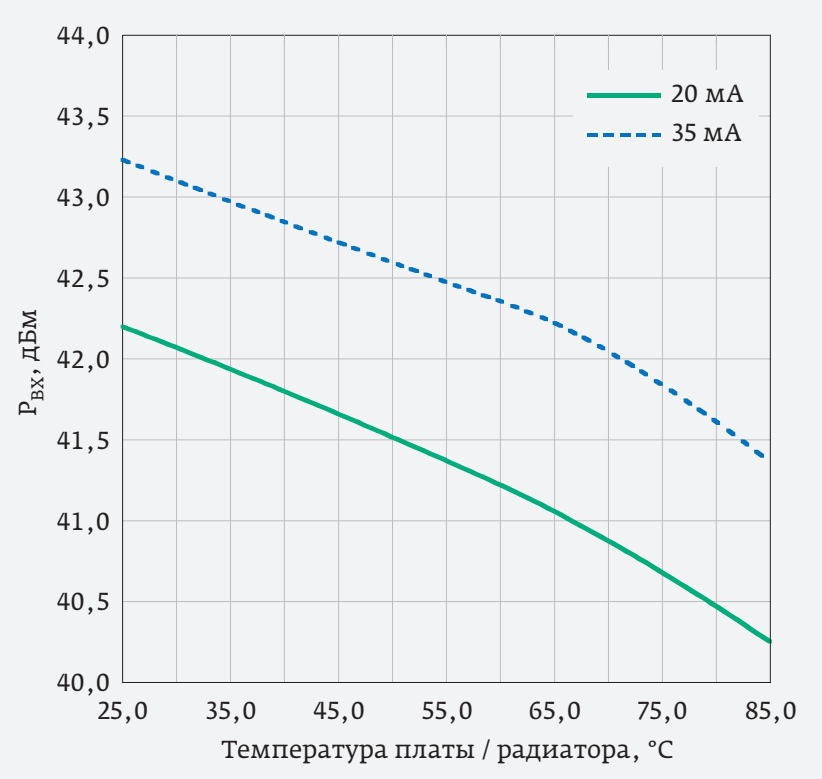

Рис. 12. Зависимость максимальной допустимой входной мощности от температуры печатной платы/радиатора при двух значениях тока смещения (модель MASW-000825-12770T, компания MACOM) 


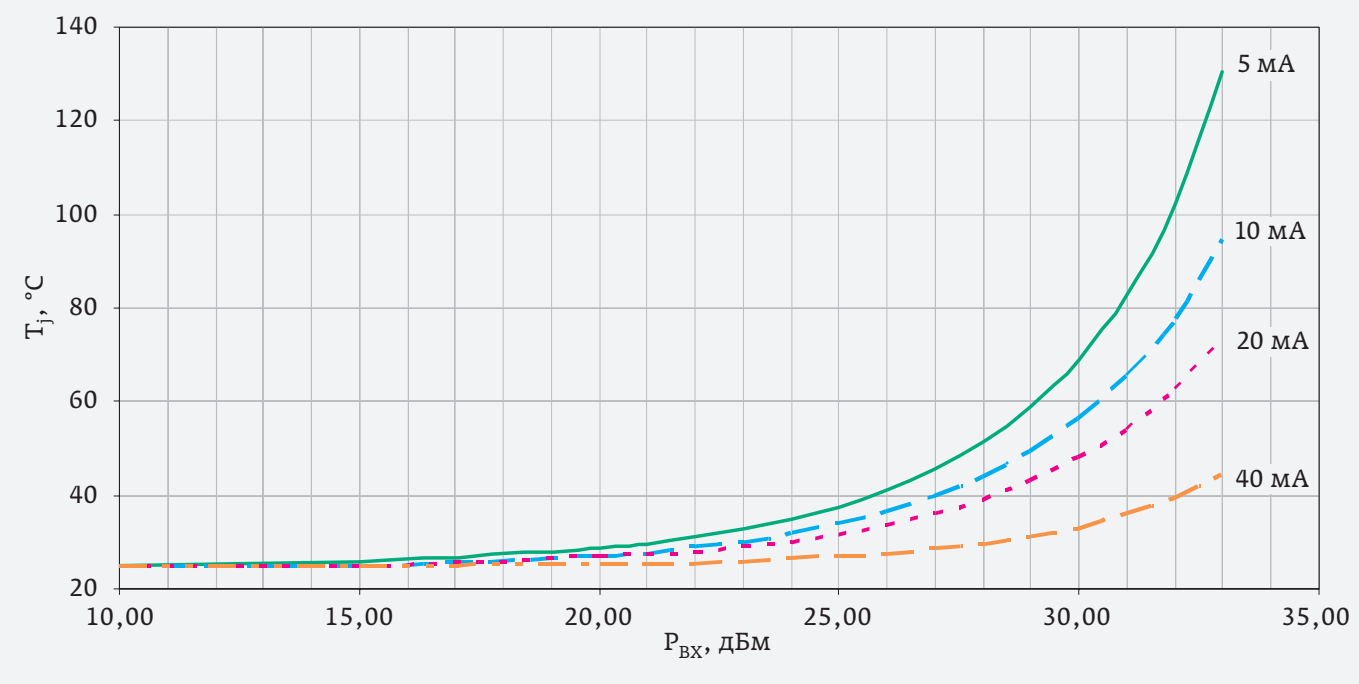

Рис. 13.

Зависимость температуры p-n-перехода от уровня входной мощности при различных токах, протекающих через диод (модель MASW-00210213580 , компания МАСОМ)

\section{Мощные pin-диодные переключатели в модульном исполнении}

Количество компаний, производящих интегральные pin-диодные переключатели, относительно невелико (MACOM, Analog Devices, Skyworks Solutions, Qorvo, Microsemi, KCB Solutions). Производителей модульных pinдиодных переключателей существенно больше (JFW, PMI, AMC, Kratos, G.T. Microwave, UMCC, RF-Lambda, Mercury Systems, ETL, Comtech, CPI, CMC, A-INFO, Corry Micronics, KORTCOM, LabTech, Narda, Linwave и др.). Многие из этих компаний производят модульные переключатели уже несколько десятилетий. И, если в прошлые годы при их производстве применялись только дискретные pin-диоды, то сейчас модульные переключатели могут быть реализованы и с использованием интегральных разработок. Большое число компаний-производителей и еще большее количество выпускаемых ими моделей (табл. 5, 6) связаны с многообразием решаемых длительное время конкретных технических задач и значительным спросом на такого рода переключатели с постоянно улучшаемыми характеристиками. Разнообразие технических характеристик свидетельствует о востребованности мощных ріп-диодных переключателей на современном рынке.

Анализ табличных данных позволяет сделать вывод о том, что наибольшие допустимые значения мощности реализуются в переключателях, предназначенных для работы на низких частотах. Так, допустимая входная мощность 1000 Вт реализована в изделиях SH90207 и SH91207, предназначенных для работы на частотах 1,5-50 МГц. Столь высокий уровень допустимой мощности обеспечен при токах порядка 1 А и обратном напряжении 700 В. Вносимые потери и развязка в этих переключателях равны 0,15 и 37 дБ соответственно

Здесь также стоит упомянуть разработанный для радиолокационного диапазона частот 150-600 МГц
SPDT Tx/Rx переключатель [17] с импульсной мощностью более 1000 Вт ( $P_{\text {ср }}=150$ Вт), который наряду с относительно низкими вносимыми потерями (1,6 дБ) обеспечивает уровень развязки более 85 дБ.

По мере повышения рабочего диапазона частот уровень входной допустимой мощности снижается и на частотах порядка 20 ГГц не превосходит единиц ватт (см. табл. 6). Есть, однако, и исключения. Так, в SPDT-переключателе Н26-206 компании Comtech коммутируемая мощность достигает 80 Вт в диапазоне частот 6-18 ГГц.

Применяемые при конструировании мощных переключателей технические решения в силу разницы допустимых значений мощности, рабочих частот, отличий, определяемых областью применения, и др. весьма разнообразны (рис. 19-21). Возможности конструкторских решений здесь чрезвычайно широки. Например, производимые компанией RF-Lambda переключатели типа SPTT (модели RFSPTTxxxxx), могут быть реализованы как минимум тремя различными способами (рис. 22). В соответствии стребованиями заказчика характеристики поставляемых изделий могут существенно отличаться от характеристик

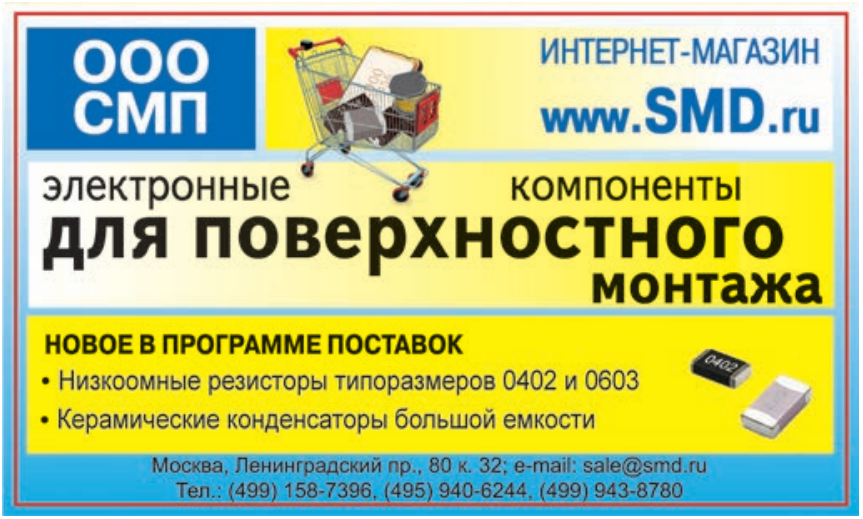


Таблица 7. Волноводные переключатели

\begin{tabular}{|c|c|c|c|c|c|c|c|c|}
\hline Компания & Серия, модель & Тип & $\begin{array}{r}\text { Диапазон } \\
\text { частот, } \\
\text { ГГц }\end{array}$ & $\begin{array}{c}\mathrm{P}_{\text {доп }}, \\
\mathrm{BT}\end{array}$ & $\begin{array}{l}P_{\text {и }}, \\
\text { Вт }\end{array}$ & $\begin{array}{l}\text { IL, } \\
\text { дБ }\end{array}$ & $\begin{array}{l}\text { Iso, } \\
\text { дБ }\end{array}$ & $\begin{array}{l}\mathrm{T}_{\text {п }}, \\
\mathrm{HC}\end{array}$ \\
\hline CPI & BLP2084 & SPDT & $9,2-9,8$ & - & 3000 & $<1$ & $>40$ & $<30000$ \\
\hline CPI & VLU2038 & SPST & $15,7-17,7$ & 20,0 & 20 & $<0,8$ & $>35$ & $<200$ \\
\hline $\begin{array}{l}\text { SAGE } \\
\text { Millimeter }\end{array}$ & SKS-5037533030-1515-R1 & SPST & $50,0-75,0$ & $<0,2$ & - & 2 & 30 & 100 \\
\hline $\begin{array}{l}\text { SAGE } \\
\text { Millimeter }\end{array}$ & SKS-9031042825-1010-R1 & SPST & $90,0-100,0$ & $<0,25$ & - & 2,8 & 25 & 100 \\
\hline $\begin{array}{l}\text { SAGE } \\
\text { Millimeter }\end{array}$ & SKS-5536536535-1515-R1 & SP4T & $55,0-65,0$ & $<0,2$ & - & 6,5 & 35 & 100 \\
\hline ELVA-1 & SPST-42 & SPST & $18,0-26,5$ & - & 1,0 & 0,7 & $>30$ & $<6$ \\
\hline ELVA-1 & SPST-15 & SPST & $50,0-75,0$ & - & 1,0 & 0,8 & $>30$ & $<6$ \\
\hline ELVA-1 & SPST-06 & SPST & $110,0-150,0$ & - & 0,8 & 1,5 & $>30$ & $<6$ \\
\hline HXI & HSWM4201 & SPST & $18,0-26,5$ & 1 & - & 1,5 & 30 & 3 \\
\hline HXI & HSWM22801 & SPDT & $26,5-40,0$ & 1 & - & 2,0 & 28 & 3 \\
\hline HXI & HSWM22201 & SPDT & $33,0-45,0$ & 1 & - & 2,4 & 27 & 3 \\
\hline HXI & HBSW94 & SPST/SPDT & $93,0-95,0$ & 10 & 1000 & 1,0 & 35 & - \\
\hline Millitech & PSP-42 & SPST & $18,0-26,5$ & 0,5 & 10 & $<2,0$ & $>40$ & 150 \\
\hline Millitech & PSP-19 & SPST & $40,0-60,0$ & 0,5 & 10 & $<2,5$ & $>25$ & 150 \\
\hline Millitech & PSP-10 & SPDT & $75,0-110,0$ & 0,5 & 10 & $<3,7$ & $>25$ & 150 \\
\hline Millitech & PSH-28 & SPST & $26,5-40,0$ & 0,25 & 5 & $<2,0$ & $>30$ & 2 \\
\hline Millitech & PSH-15 & SPST & $50,0-75,0$ & 0,25 & 5 & 2,5 & $>20$ & 2 \\
\hline Millitech & PSH-10 & SPST & $75,0-95,0$ & 0,25 & 5 & $<3,5$ & $>18$ & 2 \\
\hline Millitech & PDT-28 & SPDT & $26,5-40,0$ & 0,5 & 10 & $<2,0$ & $>22$ & 300 \\
\hline Millitech & PDT-19 & SPDT & $40,0-60,0$ & 0,5 & 10 & $<2,5$ & $>22$ & 300 \\
\hline Millitech & PDT-12 & SPDT & $60,0-90,0$ & 0,5 & 10 & $<3,0$ & $>19$ & 300 \\
\hline
\end{tabular}

стандартных моделей. Возможные значения допустимых мощностей лежат в пределах 0,2-200 Вт. Следует, однако, иметь в виду, что повышение требований к уровню допустимой входной мощности приводит к снижению уровня развязки, увеличению необходимого тока смещения и времени переключения, а за уменьшение вносимых потерь и увеличение развязок приходится платить сужением рабочего диапазона частот.

Зависимости IL и Iso от частоты, полученные для различных значений температуры окружающей среды (рис. 23), свидетельствуют о том, что изменение температуры в большей степени сказывается на величине вносимых потерь и в меньшей степени - на величине развязки.

Большинство переключателей изготавливаются в промышленном исполнении. Однако, некоторые компании специализируются на выпуске изделий для жестких условий эксплуатации. Так, например, многие переключатели компании RF-Lambda производятся в герметичном исполнении, а вся линейка изделий компании UMCС удовлетворяет требованиям военного стандарта MIL-STD-202F в диапазоне температур $-55 . .95{ }^{\circ} \mathrm{C}$. При проведении испытаний данных переключателей по этому стандарту подтверждается их влагостойкость (96 ч при 95\%-ной 


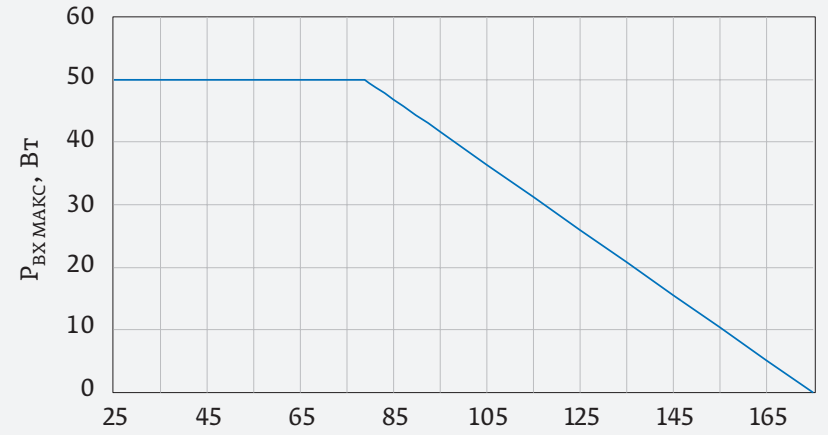

a)

Температура основания корпуса, ${ }^{\circ} \mathrm{C}$

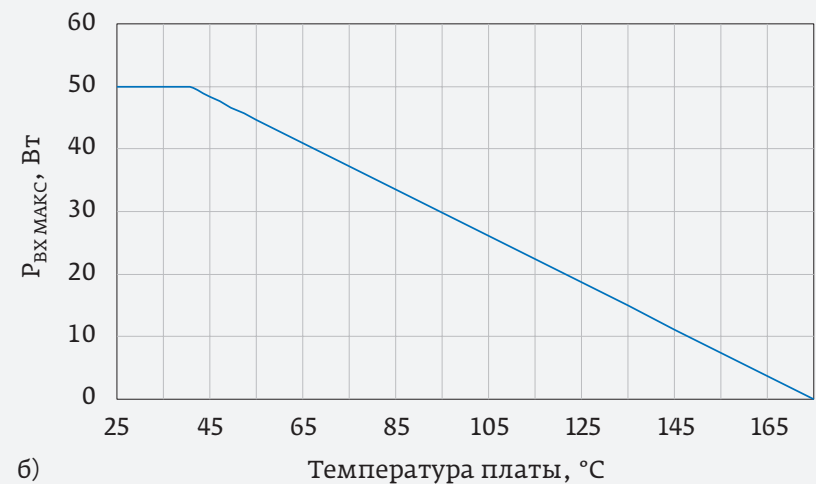

Рис. 14. Зависимости максимально допустимой мощности от температуры основания корпуса (а) и температуры печатной платы (б) (модель SKY12208-306LF, компания Skyworks Solutions)

относительной влажности), устойчивость к ударам (75 g, 6 мс), вибростойкость (15 g), стойкость к температурным шокам (пять циклов) и работоспособность на больших высотах (50000 футов). Отметим также, что многие переключатели компании Miteq (ныне L3 Narda-Miteq), также удовлетворяют требованиям военных стандартов.

\section{Волноводные СВЧ-переключатели}

Волноводные переключатели используются для изменения пути прохождения СВЧ-сигналов в целях обеспечения импульсной модуляции, защиты входных цепей приемников, коммутации антенных лучей и каналов, в переключаемых фильтрах и дуплексорах. Область использования этих переключателей чрезвычайно

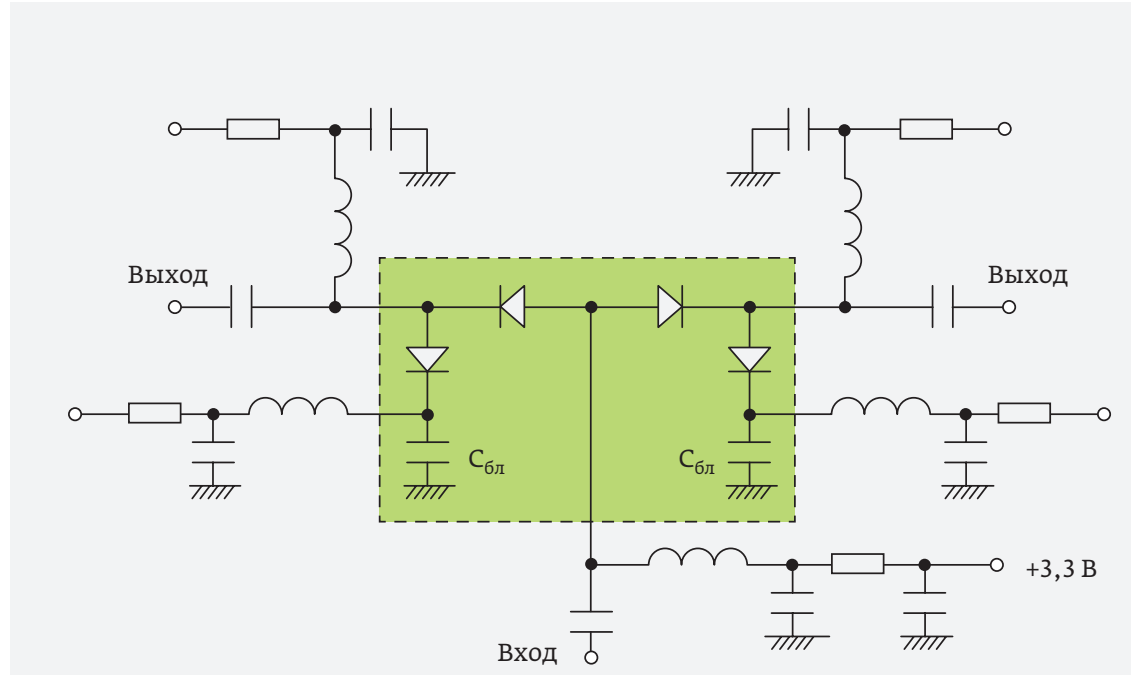

Рис. 16. Принципиальная схема SPDT-переключателя (модель KS113-52 компании KCB Solutions). Зеленым фоном выделена интегральная часть изделия

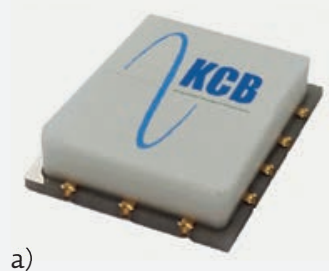

a)

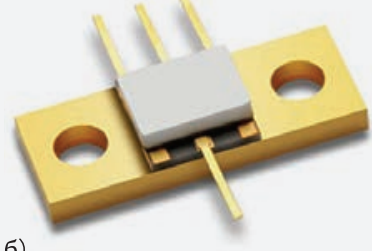

б)

Рис. 15. СВЧ-переключатели компании КСВ Solutions: а - в QFN-корпусе (модель KS102-55, 100 Вт), б - во фланцевом корпусе (модель KS03R3-22, 200 Вт)

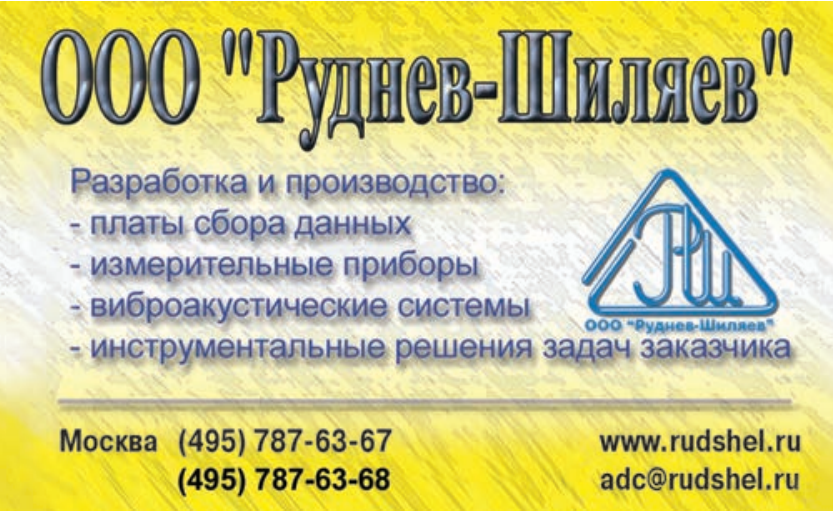




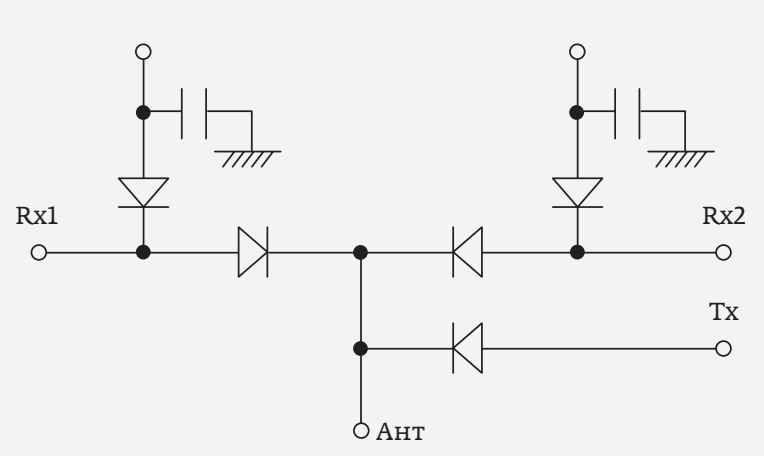

Рис. 17. Упрощенная принципиальная схема асимметричного SP3Т-переключателя компании КCB Solutions

широка. Это РЛС наземного, морского, авиационного и космического базирования, военные и коммерческие системы связи, средства радиоэлектронной борьбы, комплексы для тестирования и измерения параметров различных радиокомпонентов.

Волноводные СВЧ-переключатели в основном реализуются на кремниевых и арсенид-галлиевых ріп-диодах. Такие переключатели предлагаются на рынке многими зарубежными производителями, они рассчитаны на использование на частотах до 110 ГГц и предлагаются в различных конфигурациях, например SPST (CPI,

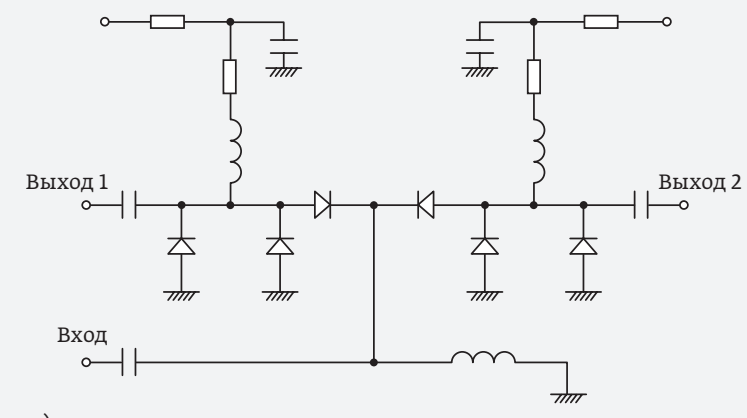

a)

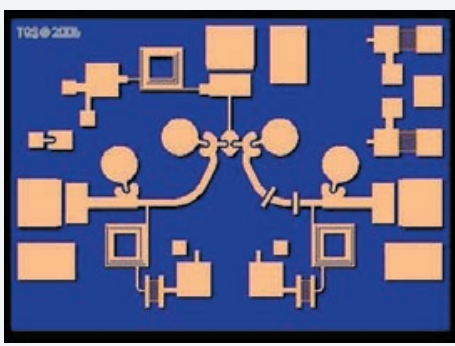

Рис. 18. Принципиальная схема (а) и топология (б) SPDT-переключателя TCS2302 компании Qorvo

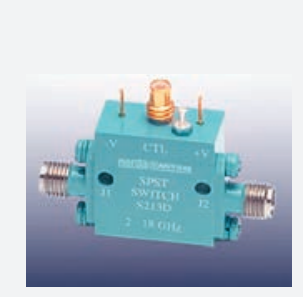

a)

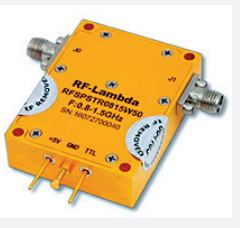

б)

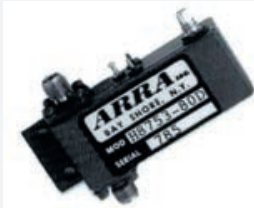

B)
Рис. 19. pin-диодные SPST-переключатели в модульном исполнении: а - модель S213D, компания Narda; 6 - модель RFSPSTR0815W50, компания RF-Lambda; в - модель H8753-80D, компания ARRA

Millitech, ELVA-1, QuinStar Technology, HXI, Fairview Microwave, Mi-Wave, SAGE Millimeter), SPDT (CPI, Millitech, HXI, Fairview Microwave, Mi-Wave, SAGE Millimeter), SP4T (SAGE Millimeter, Ducommun), SPIOT (Ducommun). Значения допустимых мощностей в этих переключателях снижаются по мере увеличения полосы рабочих частот (табл. 7), причем различия в допустимых значениях непрерывной и импульсной мощности зависят от длительности импульсов и скважности. Конструктивно эти переключатели могут также различаться и весьма существенно (рис. 24).

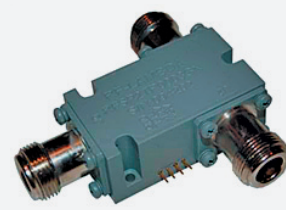

a)

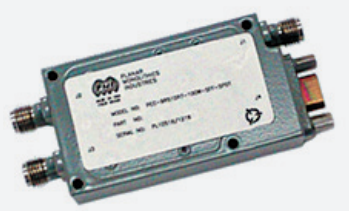

г)

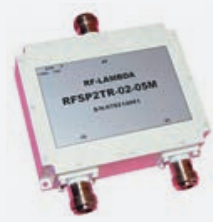

б)

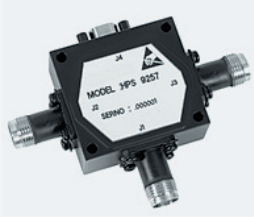

B)

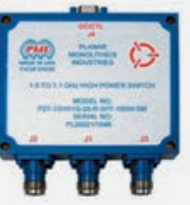

д)

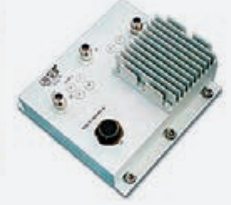

e)

Рис. 20. pin-диодные SPDT-переключатели в модульном исполнении: а - модель RFSP2TR0005M, компания RF-Lambda; 6 - модель RFSP2TR-02-05M, компания RF-Lambda; в - модель HPS-9257, компания Kratos; г - модель PEC-9R510R7-100W-SFF-SPDT, компания PMI; д - модель P2T-1G1R1G-25-R-5FF-100W, компания PMI; е - модель VLC1599, компания CPI 


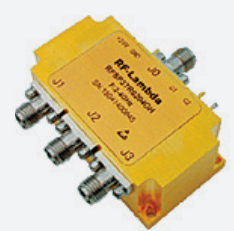

a)

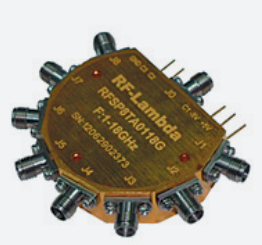

г)

б)
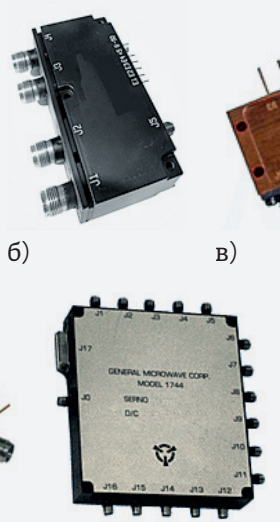

д)

B)
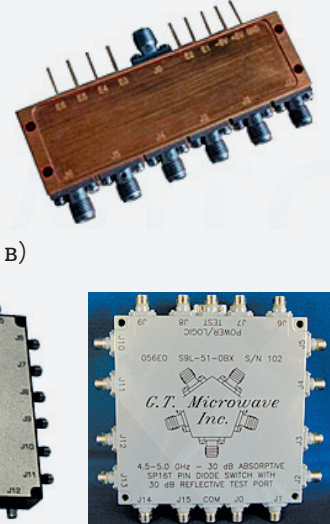

e)
Рис. 21. Многопозиционные ріп-диодные переключатели в модульном исполнении: а - модель RFSP3TR0204GH (SP3T, компания RF-Lambda); 6 - модель SW-1000-00 (SP4T, компания Kratos); в- модель SW-SP6T-C1-1604 (SP6T, компания ETL); г - модель RFSP8TA0118G (SP8T, компания RF-Lambda); д - модель 1744 (SP16T, компания Kratos); е - модель S9L-51-0BX (SP17T, компания G. T. Microwave)

Компания Millitech предлагает две серии SPST-переключателей (PSH, PSP) и одну серию SPDT-переключателей (РDT) с временами переключения 2, 150 и 300 нс для перечисленных серий соответственно. В каждой из названных серий стандартные модели переключателей могут быть изготовлены для диапазонов частот 18-26,5; 26,5-40; 33-50; 40-60; 50-75; 60-90; 75-95 ГГц, а в сериях PSP и PDT также для диапазонов 75-110 гГц и 75-100 ГГц

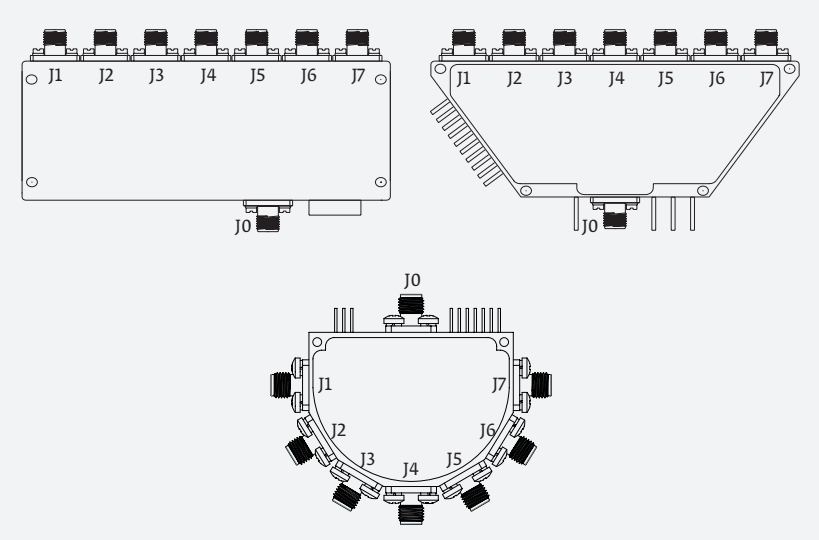

Рис. 22. Варианты конструктивного исполнения многопозиционных SP7T-переключателей компанией RF-Lambda (модели RFSP7Txxxxx)

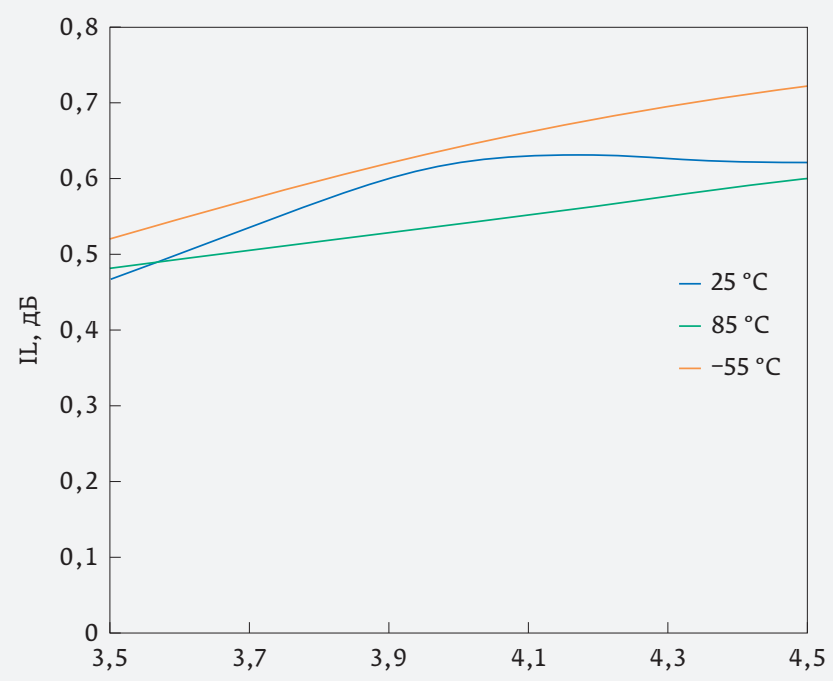

a)

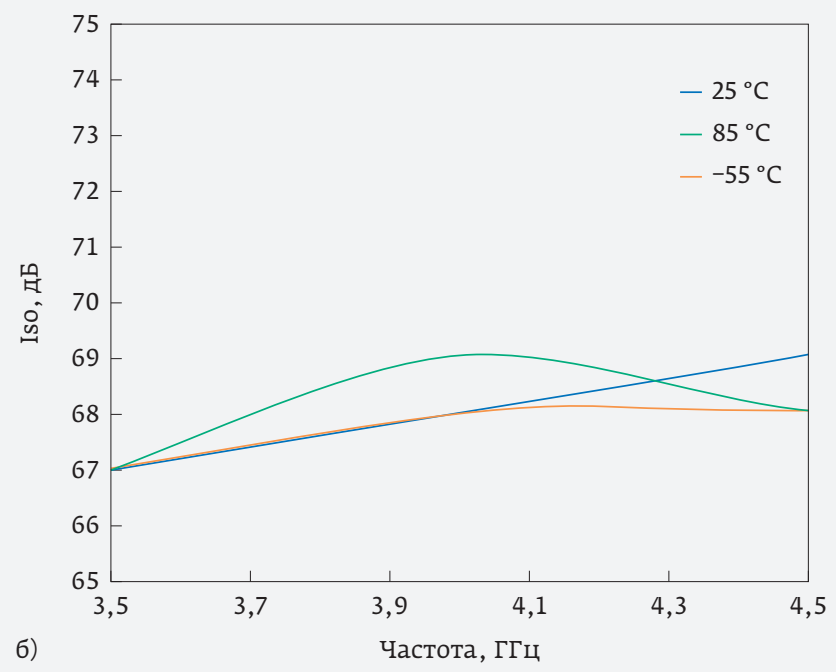

Рис. 23. Зависимости IL и Iso от частоты при различных температурах окружающей среды (модель RFSPSTR0304C, компания RF-Lambda)

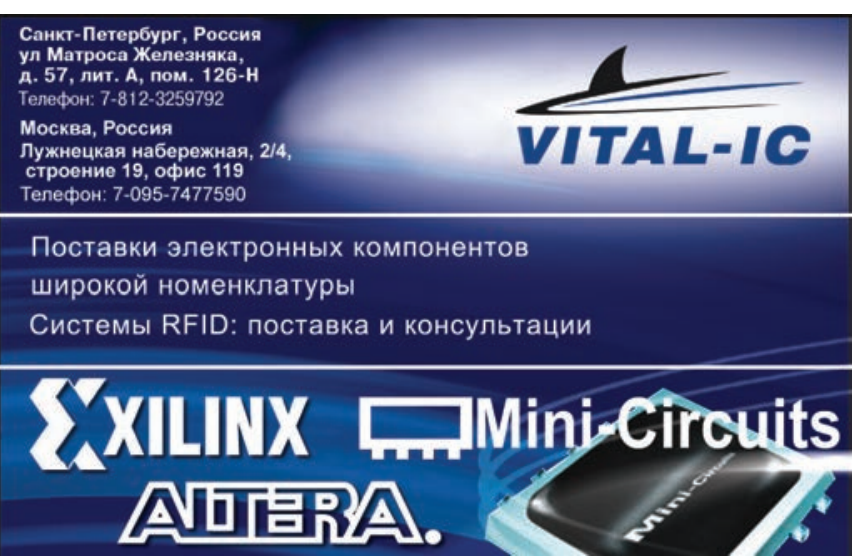




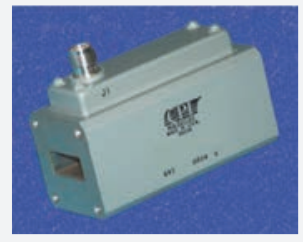

a)

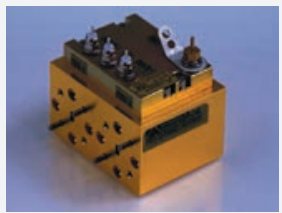

г)

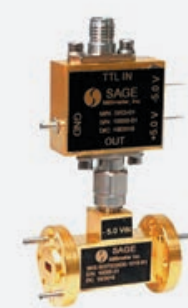

б)

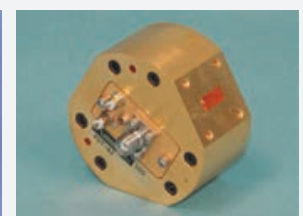

д)

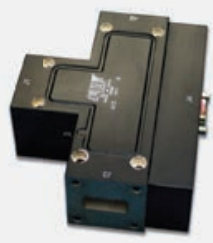

B)

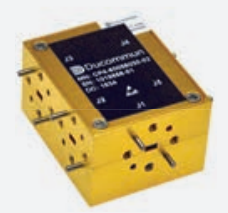

e)

Рис. 24. Волноводные pin-диодные переключатели: а - модель МА32032 (SPST, компания CPI); б - SKS-5037533030-1515-R1 (SPST, компания SAGE Millimeter); в - BLP2084 (SPDT, компания CPI); г, д - PDT-серия (SPDT, компания Millitech); е - CP4-60086030-02 (SP4T, компания Ducommun)

соответственно. Развязка между входом и выходом может быть увеличена до 60 дБ при последовательном включении через вентиль двух переключателей. Допустимые мощности в непрерывном и импульсном режимах достигают значений 0,25/5 Вт (серия PSH) и 0,5/10 Вт (серии РSР и РDT).

Компания HXI выпускает две серии SPST/SPDT-переключателей. SPST/SPDT-переключатели серии HSWM поставляются для трех диапазонов частот: 18-26,5; 26,5-40 и 33-45 ГГц. SPST-изделия серии HSW доступны для восьми диапазонов частот: 18-26,5; 22-33; 26,5-40; 33-50; 40-60; 50-75; 60-90 и 75-110 ГГц, причем в последних трех диапазонах максимально возможная полоса входных сигналов не превосходит 10 ГГц. SPDT-переключатели серии HSW выпускаются для семи диапазонов частот: 18-26,5; 26,5-40; 33-50; 40-60; 50-75; 60-90 и 75-110 ГГц. В этом случае максимально возможная полоса не превосходит 10 ГГц во всех перечисленных диапазонах. В рамках этой серии возможна поставка переключателей и с другими диапазонами рабочих частот, например: 33-37 ГГц (HSW2803-xxx), 55-65 ГГц (HSW1501-xxx), 90-98 ГГц (HSW1001-Xxx), 93-95 ГГц (HBSW94-XXX). В последнем из этих переключателей допустимая непрерывная мощность равна 10 Вт, а импульсная достигает $1000 \mathrm{BT}$.

SPST-переключатели с импульсной мощностью до 1 Вт для девяти диапазонов частот: 18-26,5; 26-40, 33-50, 40-60, 50-75, 60-90, 75-110, 90-140, 110-150 ГГц производит

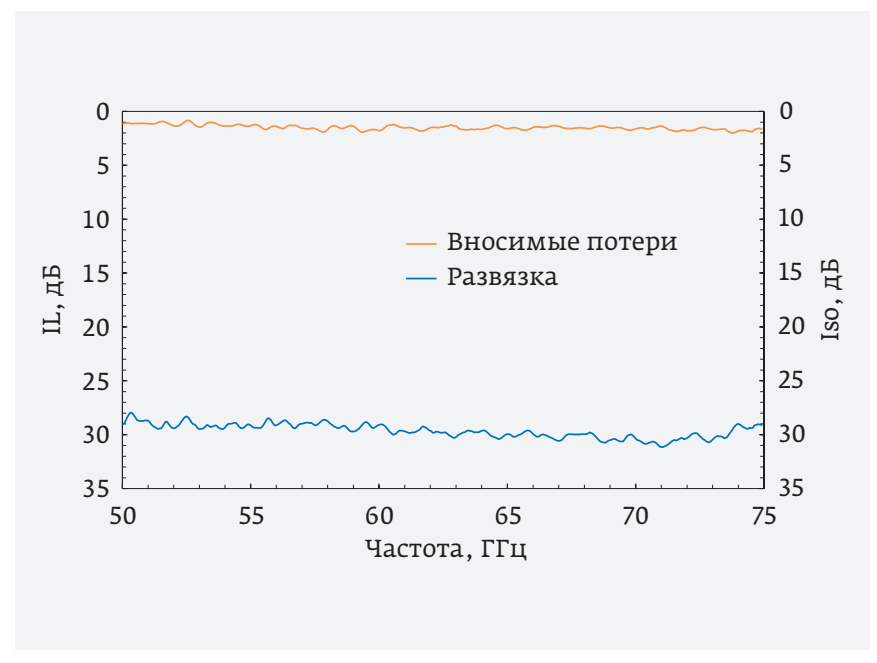

Рис. 25. Зависимости IL и Iso от частоты в волноводном pin-диодном SPST-переключателе (модель SKS-5037533030-1515-R1, компания SACE Millimeter)

компания ELVA-1. Возможная полоса рабочих частот в этих диапазонах превышает 10\% от центральной частоты диапазона.

Отражательные SPST-переключатели в стандартном исполнении выпускаются компанией SAGE Millimeter для диапазонов частот 45-55, 55-65, 75-85 и 90-100 ГГц. Кроме того, SPST-переключатели доступны для диапазонов частот 34-37; 26,5-40; 35-45; 50-75; 60-90; 75-90; 75-110 ГГц, SPDT-изделия - для диапазонов частот 55-71, 75-85, 60-90, 90-100, 75-110 ГГц, а SP4T-переключатели - для диапазонов частот 55-65, 50-75 и 60-90 ГГц. Надо отметить, что производителем обеспечиваются практически постоянные значения вносимого ослабления и развязки во всем диапазоне рабочих частот (рис. 25).

Несколько моделей волноводных переключателей выпускает компания СРІ (см. табл. 7). В одном из них (модель BLP2084), рассчитанном на работу в Х-диапазоне, импульсная мощность при длительности импульса $\mathrm{T}_{\text {и }}=40$ мкс и скважности $\mathrm{Q}=10 \%$ при холодном режиме переключения может достигать 3000 Вт.

\section{ЛИТЕРАТУРА}

15. Boles T., Brogle J., Rozbicki A. A Monolithic, 1000 Watt SPDT Switch // 2008 IEEE MTT-S International Microwave Symposium Digest. 2008. PP. 1285-1288.

16. Yang J.G., Yang K. High-Linearity K-Band AbsorptiveType MMIC Switch Using GaN PIN-Diodes // IEEE Microwave and Wireless Components Letters. 2013. V. 23, № 1, P. 37-39.

17. Rodriguez-Morales F., Gogineni S., Ahmed F., et al. T/R Switches and Modules for Ice Sounding / Imaging Radar // Microwave Journal. Aerospace \& Defense Electronics Supplement. May 2016. P. 6, 8, 10, 12, 14, 16, 18 


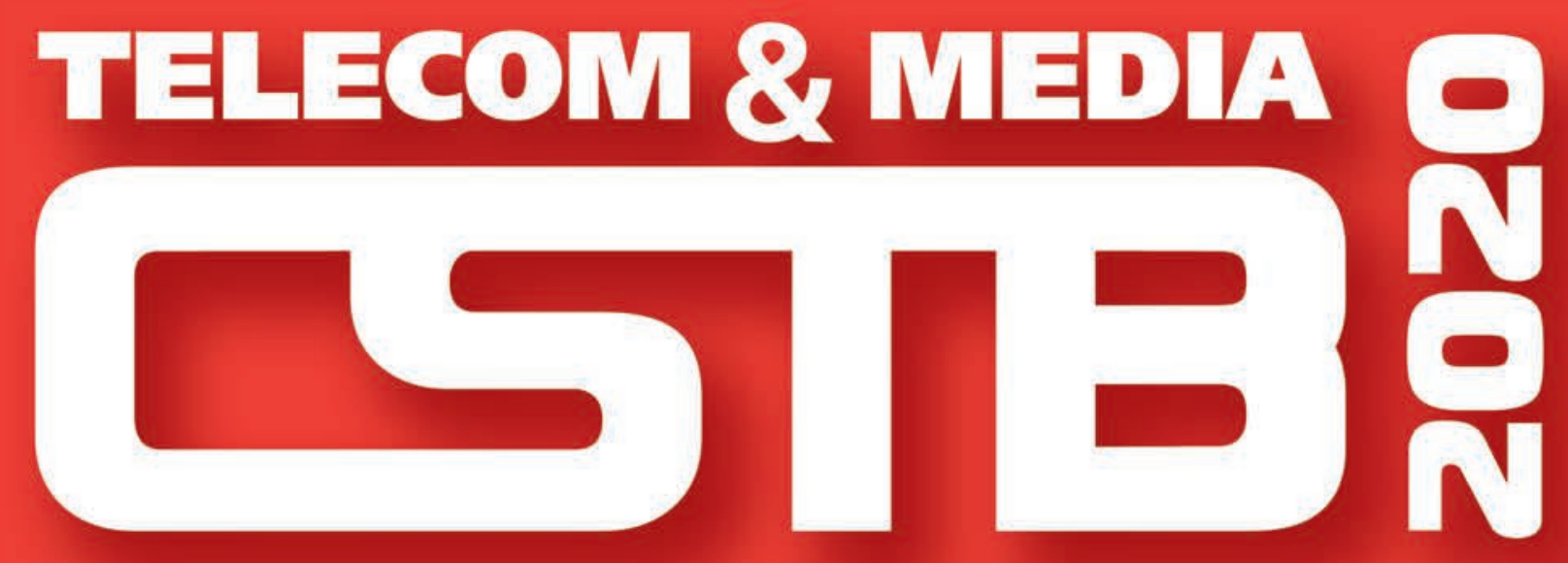

НОВЫЙ ФОРМАТ

НОВЫЕ ВОЗМОЖНОСТИ

\section{В ПРОГРАММЕ}

- Форум «Технологии и сервисы»

- Форум «Content Summit Russia»

- 11-я Национальная Премия «Большая Цифра-2020»

- Тематические экспозиции OTT.MARKET и CONTENT.MARKET

\section{CSTB.RU}

